MZ-TH/10-37

September 2013

\title{
New Sum Rule Determination of the Mass and Strangeness Content of the Nucleon
}

\author{
N.F. Nasrallah ${ }^{(a)}$ and K. Schilcher ${ }^{(b),(c)}$ \\ ${ }^{a}$ Lebanese University, Faculty of Science \\ Tripoli, Lebanon \\ (b) Institut für Physik, Johannes Gutenberg-Universität \\ Staudingerweg 7, D-55099 Mainz, Germany \\ ${ }^{(c)}$ Centre for Theoretical Physics and Astrophysics \\ University of Cape Town, Rondebosch 7700, South Africa \\ E-mail: nsrallh@ul.edu.lb, karl.schilcher@uni-mainz.de
}

\begin{abstract}
A new QCD calculation of the mass of the nucleon is presented. It makes use of a polynomial kernel in the dispersion integrals tailored to practically eliminate the contribution of the unknown $1 / 2^{+}$ and $1 / 2^{-}$continuum. This approach avoids the arbitrariness and instability attached to the Borel kernel used in previous sum rules calculations. Our method yields stable results for the nucleon mass and coupling. For standard values of the condensates, the prediction of the nucleon mass in the chiral limit is $m_{N}=(830 \pm 50) \mathrm{MeV}$. With the pion-nucleon sigma-term given by chiral perturbation theory and the strange sigma-term estimated by the Zweig rule we get $m_{N}=(990 \pm 50) \mathrm{MeV}$.
\end{abstract}




\section{Introduction}

The nucleon sigma terms are defined by

$$
\begin{gathered}
\sigma_{\pi N}=\hat{m}\langle N(p)|\bar{u} u(0)+\bar{d} d(0)| N(p)\rangle \\
\sigma_{s}=m_{s}\langle N(p)|\bar{s} s(0)| N(p)\rangle
\end{gathered}
$$

where $\hat{m}=\frac{1}{2}\left(m_{u}+m_{d}\right)$ and $m_{u}, m_{d}, m_{s}$ refer to quark masses. The sigma term was introduced in chiral perturbation theory (ChPT) to measure the explicit breaking of chiral symmetry due to non-zero masses of light quarks .It represents the contribution from the finite quark masses to the mass of the nucleon and it contains important information on the strangeness content of the nucleon and the quark mass ratios. The pion-nucleon sigma term is related to the value of the pion-nucleon invariant amplitude at the unphysical Cheng-Dashen point where $s-u=0, t=2 m^{2}$ (here, $\mathrm{s}, \mathrm{t}, \mathrm{u}$ are the Mandelstam variables). Recently in a paper on the elastic scattering of supersymmetric cold dark matter particles on nucleons it has been shown that the cross sections depend strongly on the value of the pion-nucleon sigma term $\sigma_{\pi N}$ 1]. Related to the sigma terms is the so-called strangeness content of the nucleon be defined as

$$
y \equiv 2 \frac{\langle N(p)|\bar{s} s| N(p)\rangle}{\langle N(p)|\bar{u} u+\bar{d} d| N(p)\rangle} .
$$

The quantity $y$ relates the two sigma terms,

$$
\sigma_{s}=\frac{y}{2} \frac{m_{s}}{\hat{m}} \sigma_{\pi N} .
$$

The sigma term can be calculated from Lattice Gauge Theory (LGT) using the Feynman-Hellmann theorem applied to the nucleon mass. The LGT results are rather frustrating. Predictions vary between $20 \mathrm{MeV}$ and $90 \mathrm{MeV}$, with incompatible errors (for a recent compilation of LGT results see [5]). Experimental results come from dispersion relation analysis of pion-nucleon scattering data. They are equally confusing. To quote two recent publications.:A George Washington University phase-shift analysis from 2005 leads to a sigma term of $81 \pm 6 \mathrm{MeV}$ [6]. A more recent analysis of the same TRIUMF data[7] gives $\sigma_{N}=43 \pm 12 \mathrm{MeV}$.

In view of the importance of the sigma term the situation concerning both theory and experiment is totally frustrating. We therefore hope to put some light into the issue by communicating our results of a QCD sum rule analysis of the nucleon mass which allows conclusions on the sigma terms and the strangeness content of the nucleon. The QCD sum rule method introduced by Shifman et al. 8 has extended the applicability of QCD far beyond simple perturbation theory. The method was adapted to the case of nucleons by Ioffe [2] and independently by Chung, Dosch, Kremer and Schall [9. These authors showed how to approach one of the fundamental problems of $\mathrm{QCD}$, the calculation of baryon masses from the Lagrangian and the vacuum condensates. The critical problem of previous 
calculations was the unknown couplings of the higher nucleon resonances to the nucleonic current on the hadronic side of the sum rule. Using a new sum rule approach, we obtain for the first time stable and unambiguous results for the nucleon mass in the chiral limit.

Nucleon mass sum rules start with the correlation function

$$
\Pi(q)=i \int d^{4} x e^{i q x}\langle 0|\eta(x) \eta(0)| 0\rangle
$$

where $\eta$ is a nucleon interpolating field constructed from local QCD operators with the quantum numbers of the nucleon. We will choose [2]

$$
\eta=e^{a b c}\left(u^{a} C \gamma^{\lambda} u^{b}\right) \gamma_{5} \gamma^{\lambda} d^{c} .
$$

which couple maximally to the nucleon. The correlator can be decomposed in terms of invariants,

$$
\Pi(q)=q_{\mu} \gamma^{\mu} \Pi_{1}\left(q^{2}\right)+\Pi_{2}\left(q^{2}\right)
$$

with $\gamma_{\mu}$ standing for the Dirac matrices. $\Pi\left(t=q^{2}\right)$ is an analytic function in the complex $t$-plane with a pole at $t=m_{N}^{2}$ and a cut along the positive real axis starting at $t=\left(m_{N}+m_{\pi}\right)^{2}$. The sum rule methods can be traced back to the Cauchy formula

$$
\frac{1}{2 \pi i} \oint \Pi(t) P(t) d t=-\int_{0}^{R} \frac{1}{\pi} \operatorname{Im} \Pi(t) P(t) d t
$$

where the kernel $P(t)$ is an arbitrary analytic function. The integral on the left hand side (1.h.s.) is over a circle of radius $R$. If $R$ is taken large enough, we can replace $\Pi(t)$ on the l.h.s. by it's QCD and operator product expansion (OPE) counterpart $\Pi_{Q C D}(t)$. The right hand side (r.h.s.) involves, apart from the nucleon pole, an integral over the cut, consisting of a background plus a set of nucleonic resonances. Duality means, that the OPE result on the l.h.s. of eq. 3. is equated to the hadronic contribution on the right hand side. Traditionally the integrand on the r.h.s. is approximated by the "pole plus continuum" model,

$$
\frac{1}{\pi} \operatorname{Im} \Pi\left(q^{2}\right)=\lambda^{2} \delta\left(q^{2}-m_{N}^{2}\right)+\frac{1}{\pi} \theta\left(q^{2}-W^{2}\right) \operatorname{Im} \Pi^{O P E}\left(q^{2}\right)
$$

Here $m_{N}$ is the position of the lowest lying pole with residue $\lambda_{N}$, the coupling of the current to the nucleon state

$$
\langle 0|\eta| n\rangle=\lambda \Psi,
$$

and an effective continuum threshold $W^{2}$ which is determined in the calculation and on which the results depend sensitively.

Most sum rule studies of baryonic currents invoke a Borel transform of the correlator, i.e. they use a kernel

$$
P(t)=e^{-t / M^{2}}
$$


which introduces another, more or less arbitrary, parameter providing exponential damping of the continuum(when it is small) and suppressing high dimensional vacuum condensates(when it is large). The integral over the circle of the non-perturbative part amounts to an infinite sum of terms of the form $\frac{C_{n}}{n ! M^{2 n}}$ which become important when $M$ is small. Stability has to be established under variations of the latter parameters.

The arbitrariness in the choice of the parameters $W^{2}$ and $M^{2}$ makes that the method results in an estimate rather than a calculation of the nucleon mass.

To overcome these intrinsic ambiguities we have introduced some time ago a sum rule method [13, originally called ACD, which exploits the analyticity properties of the correlator to significantly reduce, in some cases practically eliminate, the contribution of the continuum. The breakthrough in the treatment of the continuum has been the introduction of an integration kernel in the FESR tuned to suppress substantially the resonance energy region above the ground state. This approach, specially adapted to eliminate pronounced resonances, has been recently used to extract very precise values of the light quark masses [17] and condensates [18] as well as an evaluation of the neutron-proton mass difference [19]. Our approach is based on the fact, that the contribution of the continuum in the integral on the r.h.s. of eq.(3) arises mostly from the interval

$$
I=2.0 \mathrm{GeV}^{2} \leq t \leq 3.0 \mathrm{GeV}^{2}
$$

where the four nucleon resonances $N^{+}(1440), N^{-}(1535), N^{-}(1650)$ and $N^{+}(1710)$ lie. This prompts us to choose

$$
P(t)=1-a_{1} t-a_{2} t^{2}
$$

The parameters $a_{1}$ and $a_{2}$ are chosen so as to minimize the integral $\int_{2 G e V^{2}}^{3 G V^{2}}\left|P(t)^{2}\right| d t$. Numerically $a_{1}=.807 \mathrm{GeV}^{-2}$ and $a_{2}=-.160 \mathrm{GeV}^{-4}$. With this choice the relative damping over the interval $I,\left|P(t) / P\left(m_{N}^{2}\right)\right|$ does not exceed $6 \%$ (see Fig.1). The corresponding quantity in the case of exponential kernel (for $M^{2}=1.1 \mathrm{GeV}^{2}$ e.g. ) is $36 \%$ which shows that our choice, eq.(6) provides considerably better damping to the contribution of the continuum and justifies its neglect. Another advantage of our choice is that it will involve the contribution of only one higher order unknown condensate in the calculation whereas the exponential kernel involves an infinite number of these. Similar damping of the continuum is obtained for the kernel $t P(t)$. A residual model dependency is still unavoidable as inelasticity, non-resonant background and resonance interference are impossible to guess realistically. Also here our approach helps, as a constant background is eliminated by the integration kernel. Having thus minimized the contribution we will neglect it. 


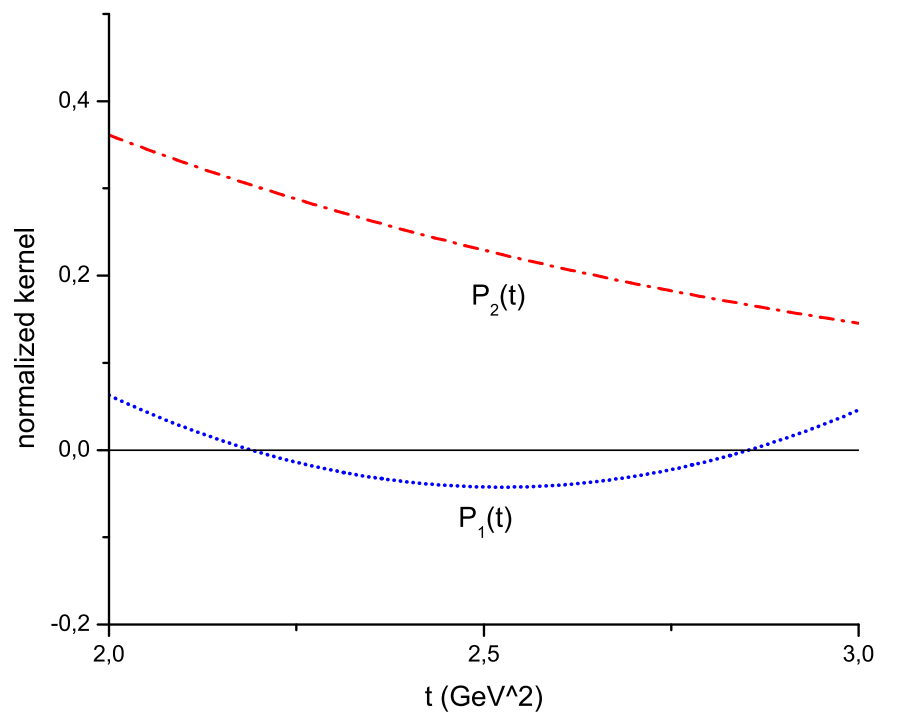

Fig. 1: $P_{1}(t)$ is our polynomial of Eq.(7) and $P_{2}(t)$ is the Borel kernel of Eq.(5). Both kernels are normalized to 1 at the nucleon mass.

The theoretical side of the sum rule, in contrast, is in better shape. The correlator (2) is known including radiative corrections and OPE terms up to dimension $\mathrm{d}=9$ [2, 10], 11]. There exists the usual uncertainty about the precise values of the condensates and the validity of the factorization assumption used for the higher dimensional condensates. We note that the kernel eq.(7) will introduce only low dimension condensates into the calculation which are known (or at least estimated).

Apart from the references cited above there are a few more attempts to evaluate the sum rule (3) involving a high sophistication on the theoretical side which is not always commensurate with the primitive model ansatz on the phenomenological side. We therefore think it necessary to present for once an (almost) model independent investigation of the nucleonic sum rule.

\section{The calculation}

The invariant amplitudes have poles at $t=m_{N}^{2}$

$$
\begin{aligned}
& \Pi_{1}(t)=\frac{-\lambda^{2}}{\left(t-m_{N}^{2}\right)}+\ldots \\
& \Pi_{2}(t)=\frac{-m_{N} \cdot \lambda^{2}}{\left(t-m_{N}^{2}\right)}+\ldots
\end{aligned}
$$


It follows then from Cauchy's theorem that

$$
\begin{aligned}
|\lambda|^{2} P\left(m_{N}^{2}\right) & =\frac{-1}{2 \pi i} \oint_{|t|=R} d t \Pi_{1}^{Q C D} P(t)+\frac{1}{\pi} \int_{t h}^{R} d t P(t) \operatorname{Im} \Pi_{1}(t) \\
m_{N}|\lambda|^{2} P\left(m_{N}^{2}\right) & =\frac{-1}{2 \pi i} \oint_{|t|=R} d t \Pi_{2}^{Q C D} P(t)+\frac{1}{\pi} \int_{t h}^{R} d t P(t) \operatorname{Im} \Pi_{2}(t)
\end{aligned}
$$

with

$$
\begin{aligned}
(2 \pi)^{4} \Pi_{1}^{Q C D}(t) & =A_{0} t^{2} \ln \frac{-t}{\mu^{2}}+A_{01} t^{2}\left(\ln \frac{-t}{\mu^{2}}\right)^{2}+A_{4} \ln \frac{-t}{\mu^{2}} \\
& +A_{6} \frac{1}{t}++A_{61} \frac{1}{t} \ln \frac{-t}{\mu^{2}}+A_{8} \frac{1}{t^{2}}+\ldots
\end{aligned}
$$

and

$$
(2 \pi)^{4} \Pi_{2}^{Q C D}(t)=B_{3} t \ln \frac{-t}{\mu^{2}}+B_{7} \frac{1}{t}+B_{9} \frac{1}{t^{2}}+\ldots
$$

The coefficients $A_{i}$ and $B_{i}$ are defined as in [11] but for a factor $(2 \pi)^{4}$ and powers of $t$ taken explicitly

$$
\begin{aligned}
& A_{0}=-\frac{1}{4}\left(1+\frac{71}{12} a\right), A_{01}=\frac{a}{8} \\
& A_{4}=-\frac{\pi^{2}}{2}\langle a G G\rangle \\
& A_{6}=-\frac{2}{3}(2 \pi)^{4}\langle\bar{q} q \bar{q} q\rangle\left(1-\frac{5}{6} a\right), A_{61}=\frac{2}{9}(2 \pi)^{4}\langle q q q q\rangle a \\
& A_{8}=\frac{-1}{6}(2 \pi)^{4} \mu_{0}^{2}\langle\bar{q} q \bar{q} q\rangle \\
& B_{3}=4 \pi^{2}\langle\bar{q} q\rangle\left(1+\frac{3}{2} a\right) \\
& B_{7}=-\frac{4 \pi^{4}}{3}\langle\bar{q} q\rangle\langle a G G\rangle \\
& B_{9}=-(2 \pi)^{6} \frac{136}{81} a\left\langle(\bar{q} q)^{3}\right\rangle
\end{aligned}
$$

where $a=\frac{\alpha_{s}\left(\mu^{2}\right)}{\pi}$. The terms $B_{7}$ and $B_{9}$ are given in the factorization approximation. In $A_{8}$ we have taken

$$
\left\langle 0\left|\bar{q} q \bar{q} a G_{\mu \nu}^{c} \frac{\lambda_{c}}{2} \sigma^{\mu \nu} q\right| 0\right\rangle=\mu_{0}^{2}\langle\bar{q} q \bar{q} q\rangle
$$

with the parameter $\mu_{0}^{2}=0.8 G e V^{2}$ as advocated in 22 .. The question at which scale this relation holds is resolved by allowing for generous errors. To avoid the 
double counting, we keep the logarithmic $\ln \left(-t / \mu^{2}\right)$ contribution in the polarization operator but neglect its anomalous dimension. In any case anomalous dimension effects are very small [12].

The contribution of the radiative corrections $A_{01}$ and $A_{06}$ turn out to be smaller than the estimated errors so we discard them for simplicity.

For the finite energy sum rule eq.(3), we need the known integrals of the form

$$
I_{i k}=\frac{1}{2 \pi i} \oint d t t^{i}(\ln (-t))^{k}
$$

These are given in convenient form in $[18$.

With our choice of $P(t)$, we then get

$$
\begin{gathered}
(2 \pi)^{4}|\lambda|^{2} P\left(m_{N}^{2}\right)=-A_{0} I_{2}(R)-A_{4} I_{0}(R)-A_{6}+a_{1} A_{8}+\Delta_{1} \\
(2 \pi)^{4}|\lambda|^{2} m_{N} P\left(m_{N}^{2}\right)=-B_{3} I_{1}(R)-B_{7}+a_{1} B_{9}+\Delta_{2}
\end{gathered}
$$

where

$$
I_{n}(R)=\int_{0}^{R} d t t^{n} P(t)
$$

and

$$
\begin{aligned}
& \Delta_{1}=-(2 \pi)^{4} \int_{t h r}^{R} d t P(t) \operatorname{Im} \Pi_{1}(t)+a_{2} A_{10} \\
& \Delta_{2}=-(2 \pi)^{4} \int_{t h r}^{R} d t P(t) \operatorname{Im} \Pi_{2}(t)+a_{2} B_{11}
\end{aligned}
$$

$A_{10}$ and $B_{11}$ are higher dimensional condensates. The integrals appearing in the expressions of $\Delta_{1}$ and $\Delta_{2}$ as well as the higher order condensates are unknown. Whereas it is possible to assess the latter using the method of Padé approximants the former are impossible to estimate in practice. The only thing we can do is minimize these integrals and then neglect them.

The choice of the function $P(t)$ aims at reducing this contribution as much as possible in order to allow its neglect. This is achieved by minimizing $P(t)$ over the resonance region. In the vast domain of QCD sum rules the usual choice would be $P(t)=\exp \left(-t / M^{2}\right)$ where the magnitude of $M$ (the Borel mass) determines the strength of the damping of the contribution of the continuum. If $M$ is small the damping is good but the contribution of the unknown terms in the QCD asymptotic expansion of the amplitudes increases rapidly. If $M$ increases the contribution of the unknown terms decreases but the damping worsens. An intermediate value of $M$ has to be chosen from stability conditions which are not met in the nucleon problem.

More information can be obtained if one uses $t P(t)$ as an integration kernel. Here however one has to verify the validity of the neglect of the unknown 
integrals which enter in $\Delta_{1,2}$ because the relative damping $\left(t P(t) / m_{N}^{2} P\left(m_{N}^{2}\right)\right)$ worsens. This quantity is still small in our case whereas damping practically disappears in the case of the exponential kernel.

The value of $R$ should not be too small as this will invalidate the use of the OPE on the circle, nor should it be too large as the kernel will start enhancing the contribution of the continuum instead of suppressing it. An intermediate value of $R$ around which the sum rules are stable should be chosen.

We then get

$$
\begin{gathered}
(2 \pi)^{4}|\lambda|^{2} m_{N}^{2} P\left(m_{N}^{2}\right)=-A_{0} I_{3}(R)-A_{4} I_{1}(R)+A_{8}+\Delta_{1}^{\prime} \\
(2 \pi)^{4}|\lambda|^{2} m_{N}^{3} P\left(m_{N}^{2}\right)=-B_{3} I_{2}(R)-B_{9}+\Delta_{2}^{\prime},
\end{gathered}
$$

where

$$
\begin{aligned}
& \Delta_{1}^{\prime}=-(2 \pi)^{4} \int_{t h r}^{R} d t t P(t) \operatorname{Im} \Pi_{1}(t)+a_{1} A_{10}+A_{12} \\
& \Delta_{2}^{\prime}=-(2 \pi)^{4} \int_{t h r}^{R} d t t P(t) \operatorname{Im} \Pi_{2}(t)+a_{1} B_{11}+B_{13}
\end{aligned}
$$

The nucleon mass can be determined by taking various ratios between eqs. (10) to (14). Using $\langle\alpha G G\rangle=.012 \mathrm{GeV}^{4},\langle q q\rangle=-(1.90 \pm 0.14) \times 10^{-2} \mathrm{GeV}^{3}$ at $\mu=2 G e V$ [17] $\alpha_{s}\left(m_{\tau}^{2}\right)=0.329 \pm 0130$ [23] and neglecting the $\Delta^{\prime} s$ we obtain three expressions for the nucleon mass

$$
\begin{gathered}
m_{N}^{2}=\left(A_{0} I_{3}(R)+A_{4} I_{1}(R)+A_{8}\right) /\left(A_{0} I_{2}(R)+A_{4} I_{0}(R)+A_{6}-a_{1} A_{8}\right. \\
m_{N}^{2}=\left(B_{3} I_{2}(R)+B_{9}\right) /\left(B_{3} I_{1}(R)+B_{7}-a_{1} B_{9}\right) \\
m_{N}=\left(B_{3} I_{1}(R)+B_{7}-a_{1} B_{9}\right) /\left(A_{0} I_{2}(R)+A_{4} I_{0}(R)+A_{6}-a_{1} A_{8}\right.
\end{gathered}
$$

At this point it is worth assessing the reliability of eqs.(10) and (11) (and consequently of eqs.(13) and (14) (19). It can be argued [20 that eq.(11) is more reliable than eq.(10) because the first order radiative correction to $A_{0}$ is anomalously large which casts doubt on the validity of the QCD expansion. Another reason is that the dominant term is provided by $A_{6}$ proportional to the 4-quark condensate the deviation of the value of which from the one given by factorization being really unknown. We therefore consider eq.(16) to be the most reliable of the three equations above and adopt it in our analysis. Of course factorization has still to be used to evaluate $B_{7}$ and $B_{9}$ but these are now non -dominant terms.

Both numerator and denominator of eq.(16) are stable in the interval $2<$ $t<3$ (see Fig.2) and so is the resulting value of the mass. 


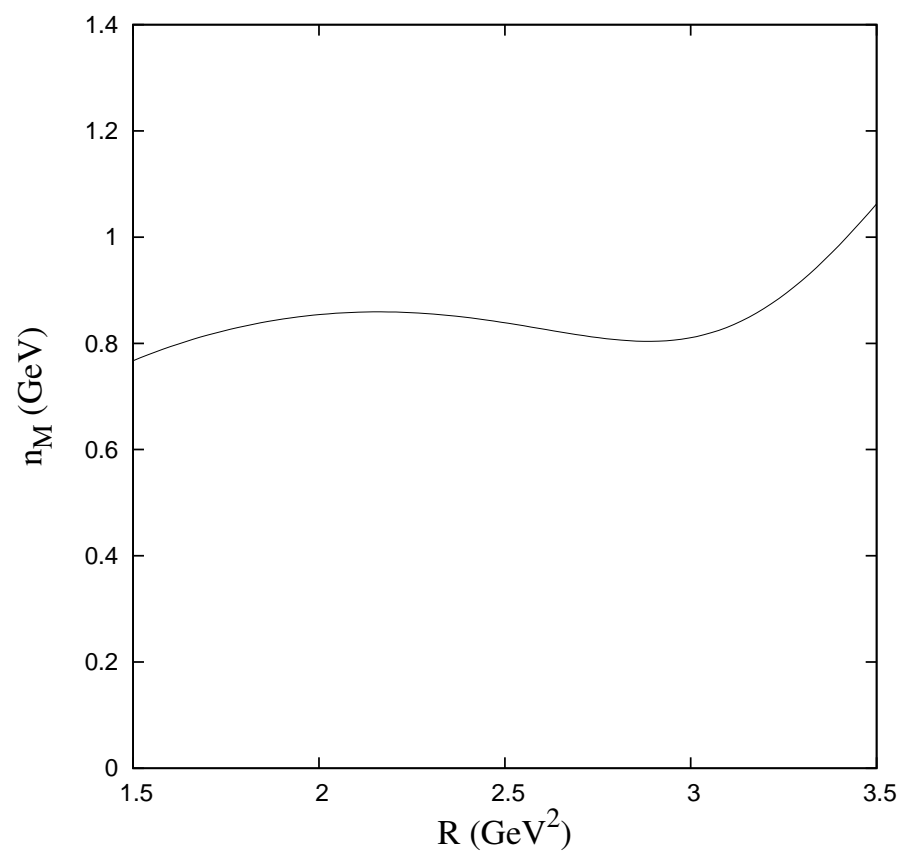

Using standard values for the condensates eq.(16) then yields

$$
m_{N}=(830 \pm 50) M e V
$$

The error having been obtained by estimating the higher order condensates using Padé approximants and by varying the coefficients $a_{1}$ and $a_{2}$ within reasonable limits in order to deplete the contribution of the continuum to the dispersion integrals.

If we use eqs. (15) and (16) nevertheless with the standard value for $A_{0}$ and with $A_{6}$ obtained from factorization we get

$$
m_{N}=810 \mathrm{MeV}
$$

and

$$
m_{N}=870 M e V
$$

respectively.

The fact that all three ratios give almost the same answer pleads for the choice of the standard values of the condensates.and for the smallness of the contribution of instantons which we have neglected. An interesting study of the effects of instantons is undertaken in 21. The damping ratio=value of kernel at resonance/value of kernel at nucleon shows that we have it much better than exponential damping which in addition cannot take moments (integral of $t P(t)$ ) and is not even stable. 


\section{Results and conclusions}

With standard values of the condensates and using the factorization approximation as discussed above, we get a value for the nucleon mass $m_{N}=(830 \pm$ 50) $\mathrm{MeV}$ close to the experimental value. For a more precise statement, we need do discuss possible errors in the sum rules. It is important to distinguish two kinds of errors, theoretical and experimental. A theoretical error of $\pm .03 \mathrm{GeV}$ arises from the uncertainty in the condensates. We used $\langle\alpha G G\rangle=.012 \mathrm{GeV}^{4}$, $\langle q q\rangle=-(1.90 \pm 0.14) \times 10^{-2} \mathrm{GeV}^{3}$ at $\mu=2 \mathrm{GeV}$ corresponding to the limits set by $m_{u}+m_{d}=(8.2 \pm 0.6) \mathrm{MeV}$ (at scale $\mu=2 \mathrm{GeV}$ ) [17] in the GMOR relation. Varying the scale parameter $\mu^{2}$ between $4 \mathrm{GeV}^{2}$ and $2 \mathrm{GeV}^{2}$ introduces an additional error of $\pm 0.03 \mathrm{GeV}$. Furthermore there is an error due to the strong coupling constant $\alpha_{s}\left(m_{\tau}^{2}\right)=0.329 \pm 013$ [23] which leads to an error of $\pm 0.6 \mathrm{MeV}$. The total error in the calculated nucleon mass is therefore mainly due to the method, i. e. due to the fact that the continuum is not completely eliminated by our method.

Noting that our nucleon mass $m_{N}$ is obtained in the chiral limit, we get for the total nucleon mass, which we denote by $M_{N}$

$$
M_{N}=m_{N}+\sigma_{\pi N}+\sigma_{s}
$$

To get some qualitative conclusions we assume that the strangeness content of the nucleon can be estimated by the Zweig rule (or large $N_{C}$ ) to be $y=0.2$. If we use this result and additional theoretical prejudice,

$$
\frac{m_{s}}{\hat{m}} \sim 25, \quad \sigma_{\pi N} \sim 45 \mathrm{MeV} \text { Chiral Perturbation Theory 24] }
$$

we can obtain an estimate for the strange sigma term

$$
\sigma_{s}=\frac{y}{2} \frac{m_{s}}{\hat{m}} \sigma_{\pi N}=2.5 \sigma_{\pi N}=113 \mathrm{MeV}
$$

which leads then to the final prediction of the nucleon mass

$$
M_{N}=990 \pm 50 \mathrm{MeV}
$$

with additional systematic errors arising from the uncertainties in the sigma terms. Although errors are large, our result for the nucleon strongly prefers a relatively small sigma term $\sigma_{\pi N}$ of order $45 \mathrm{MeV}$. There is also strong indication that the strange content of the nucleon should be somewhat smaller than $y=$ 0.2 .

Alternatively we could use our result for $m_{N}$ together with eq.(11) and a given nucleon sigma term to extract the strangeness content of the nucleon. We find

$$
\begin{array}{cl}
\text { For } \sigma_{\pi N}=45 \mathrm{MeV} & \Rightarrow y=0.11 \\
\text { For } \sigma_{\pi N}=80 \mathrm{MeV} & \Rightarrow y=.03
\end{array}
$$

In conclusion, we have presented a sum rule calculation of the nucleon mass in the chiral limit using a kernel in the dispersion integral tailored to minimize 
the contribution of the unknown continuum without involving the higher order unknown condensates. Using standard values of the known condensates and the sigma terms, we obtain for $M_{N}$ a value which agrees quite well with the

experimental one and which excludes a large value of the strageness content of the nucleon.

Acknowledgement:

This work was started when the authors were visiting the American University of Beirut, Lebanon. We like to thank the Physics Department for its hospitality.

\section{Bibliography}

\section{References}

[1] Ellis et al.Phys. Rev. D77(2008), 065026v, Nucl. Phys. B147 (1979) 385, 448

[2] B.L. Ioffe, Nucl. Phys. B188 (1981) 317 (E: B1

[3] T. P. Cheng. Phys. Rev. D13 (1976),21 61

[4] J. Beringer et al. (Particle Data Group) Phys. Rev. D86(2012), 010001

[5] G. S. Bali. et al. Nucl.Phys. B866 (2013), 1

[6] G. E. Hite, W. B. Kaufmann, and R. J. Jacob, Phys. Rev. C71(2005), 065201

[7] [J. Stahov, H. Clement, G. J. Wagner, arXiv:1211.1148]

[8] M.A. Shifman, A.I. Vainshtein and V.I. Zakharo91 (1981) 591); B.L. Ioffe and A.V. Smilga, Nuclear Physics B232 (1984) 109

[9] Y. Chung, H.G. Dosch, M. Kremer and D. Schall, Phys. Lett. 102B (1981) 175; Y. Chung, H.G. Dosch, M. Kremer and D. Schall, Nucl. Phys. B197 (1982) 55

[10] M. Jamin, Z. Phys. C 37(1988) 635; A.A. Ovchinnikov, A.A. Pivovarov and L.R. Surguladze, Int. J. Mod. Phys. A6 (1991) 2025.

[11] V. A. Sadovnikova, E. G. Drukarev, and M. G. Ryskin, Physical Review, D $72(2005) 114015$

[12] A.A. Pivovarov and L.R. Surguladze, Nuclear Physics B360 (1991)

[13] N.F. Nasrallah, N.A. Papadopoulos and K. Schilcher. Phys. Lett. B 113 (1982) 61

[14] J. Beringer et al. [Particle Data Group Collaboration], Phys. Rev. D86 (2012) 010001. 
[15] D. Espriu, P. Pascual and R. Tarrach, Nuclear Physics B214 (1983) 285; N.V. Krasnikov, A.A. Pivovarov, N.N. Tavkhelidze, Z. Phys. C19 (1983) 301 ; A.G.Oganesian, ArXiv:hep-ph/0308289 (2003)

[16] X.Jin and J.Tang ,Physical Review, D 7256,515 (1997)

[17] C.A. Dominguez, N.F. Nasrallah, R.H. Rontsch, K. Schilcher, Phys.Rev.D79:014009,2009

[18] J. Bordes, C.A. Dominguez, P. Moodley, J. Penarrocha, K. Schilcher, JHEP 1005:064,2010

[19] N. F. Nasrallah, Phys.Rev. C87,055203 (2013)

[20] D. B. Leinweber, Annals of Physics 524,328 (1997)

[21] Hilmar Forkel and Marina Nielsen, PRD55,1471 (1996)

[22] V. M. Belyaev and B. L. Ioffe, Zh. Eksp. Teor. Fiz. 83, 876, (1982).

[23] A. Pich, arXiv:1303.2262 (2013)

[24] J. Gasser, H. Leutwyler, and M. Sainio, Phys.Lett. B253, 252 (1991); B. Borasoy and U.-G. Meissner, Annals Phys. 254, 192 (1997), hep-ph/9607432. 